\title{
Focus. Exogenèse, endogenèse photographiques
}

\section{Monique Sicard}

\section{(2) OpenEdition}

\section{Journals}

Édition électronique

URL : https://journals.openedition.org/genesis/5678

DOI : 10.4000/genesis.5678

ISSN : 2268-1590

\section{Éditeur :}

Presses universitaires de Paris Sorbonne (PUPS), Société internationale de génétique artistique littéraire et scientifique (SIGALES)

\section{Édition imprimée}

Date de publication : 15 décembre 2020

Pagination : 79-84

ISBN : 979-10-231-0704-3

ISSN : 1167-5101

\section{Référence électronique}

Monique Sicard, «Focus. Exogenèse, endogenèse photographiques », Genesis [En ligne], 51 | 2020, mis en ligne le 21 décembre 2021, consulté le 07 février 2022. URL : http://journals.openedition.org/ genesis/5678; DOI : https://doi.org/10.4000/genesis.5678 


\section{Exogenèse, endogenèse photographiques}

Monique Sicard

$\mathrm{L}$ a photographie semble, chaque fois, prélever un fragment du monde. Elle apparaît ainsi, communément, porteuse d'une forte indicialité ${ }^{1}$, marquée directement par ses objets, se confondant même avec eux. Face à une photographie de la tour Eiffel, on s'exclamera ainsi volontiers : «Voilà la tour Eiffel!»

Les travaux de Rosalind Krauss ${ }^{2}$ sur le photographique l'ont conduite à écrire non seulement sur l'indicialité photographique, mais également sur les conditions indicielles auxquelles la photographie a soumis le monde clos des arts plastiques. L'urinoir de Duchamp reste emblématique d'une telle incorporation du monde réel dans l'œuvre artistique. Dix ans avant Le Photographique de Rosalind Krauss, le Çà a été de Roland Barthes adhérait déjà à cette lignée sémiotique de l'empreinte, de la trace, de l'indice. Le fait que la surface sensible du papier, de la plaque de verre, du métal, de la pellicule... soit directement modifiée par les rayonnements électromagnétiques (la lumière) émis ou réfléchis par l'objet photographié confère à la photographie un statut littéralement exogénétique. Jean-Marie Schaeffer a fondé sur cette théorie de l'empreinte l'écriture de son livre L'Image précaire 3.

Dans l'imaginaire collectif de la photographie, l'exogenèse - désignant les apports extérieurs dans la genèse photographique - semble primer sur l'endogenèse, laissant loin derrière les notions d' '«auteur» ou d' «artiste». «La photographie, c'est la nature qui parle d'elle-même!» s'exclamaient, enthousiastes, les scientifiques pionniers des années 1840, prônant même la disparition de l'observateur, jugé trop imparfait. Ce caractère exogénétique et automatique, d'où l'intervention humaine semble absente, a contribué à conférer à la photographie une forme d'objectivité, lui faisant jouer volontiers le rôle de preuve.

De fait, la photographie, née dans les années 1830, a fait émerger un espace remarquable, jusqu'alors inconnu, dont le sujet semble exclu. Cette irruption exogénétique a contaminé les domaines visuels, artistiques et littéraires, jusqu'à la crise de la représentation contemporaine, quand le réel, ne cessant de prospérer, génère une fascinante ivresse et se substitue à la représentation. La photographie même est parfois marquée d'une sur-indicialité. Ainsi les tirages photographiques de Robert Frank d'un Beyrouth détruit par la guerre civile sont-ils marqués d'éclaboussures de sang.

\footnotetext{
1. Le mot «indice» se réfère ici au sens que lui accorde le philosophe Charles Sanders Peirce (1839-1914) : «Un indice est un signe qui fait référence à l'Objet qu'il dénote en vertu du fait qu'il est réellement affecté par cet Objet.» Charles Sanders Peirce, Écrits sur le signe, trad. G. Deledalle, Paris, Seuil, coll. «L'ordre philosophique», 1978.

2. Rosalind Krauss, Le Photographique. Pour une théorie des écarts, trad. M. Bloch, A. Hindry, J. Kempf, Paris, Macula, 1990

3. Jean-Marie Schaeffer, L'Image précaire. Du dispositif photographique, Paris, Seuil, 1987.
} 


\section{Un métissage}

\section{Une écriture fictionnelle}

L'acte photographique, cependant, n'est jamais réductible à l'incorporation directe, automatique, de la réalité. Ce sont des gestes d'écriture qui, précisément, lui donnent sens ${ }^{4}$. Ils concernent le choix opportuniste d'un lieu, d'une lumière, d'un climat, d'un milieu, d'une mise en scène, d'un mouvement, d'un geste... L'écriture photographique transite par des déplacements et positionnements de l'auteur photographe, des éclairages ou des choix de lumière, des réglages techniques; ainsi, lors de la prise de vue, le choix du point de vue, du cadre, de la focale, de la vitesse, de la profondeur de champ... - et de bien plus de paramètres encore en photographie numérique - reste fondateur. Les gestes et le corps de l'opérateur s'adaptent en permanence à un environnement dans un métissage subtil entre exogenèse et endogenèse ; entre l'empreinte et le style propre de l'opérateur.

Lors des travaux de laboratoire, qu'ils soient effectués par le photographe lui-même ou par un tireur professionnel, l'endogenèse prend le pas. En photographie argentique classique, le tireur travaille seul, dans le noir, à peine éclairé par une lampe inactinique, porte soigneusement close. Ainsi l'endogenèse domine lors du développement des négatifs, lors du tirage des positifs où le geste, le savoir-faire, le caractère et l'expérience du tireur, le choix et le dosage des produits, la qualité des papiers, la sensibilité, sont des garants de la douceur, du contraste, de la luminosité et de la profondeur de la photographie finale; lors même de la retouche sur les logiciels adaptés en photographie numérique.

L'alternance régulière entre le travail en pleine lumière et l'activité d'obscurité, entre le mouvement et la quasi-immobilité, caractérise la création photographique. Il faut reconnaître qu'après des heures ou des jours consacrés au travail d'atelier, chimique ou numérique, les objets du monde, les paysages, les humains n'en paraissent que plus fascinants. L'acuité du regard, le désir de voyage sont stimulés. L'exogenèse reprend vigueur.

Il est possible de parler de «texte photographique» tant un récit semble émaner d'un enchevêtrement des images réalisées au cours des années par un même artiste. Mais ici, contrairement au texte littéraire, classiquement reçu comme pétri d'endogenèse, l'auteur a du mal à exister. Il faut attendre les années 1970 pour qu'une galerie d'art soit consacrée, en France, à la photographie; et les années 1980 pour voir pleinement reconnus les auteurs photographes. De nos jours encore, la part auctoriale n'est manifeste que pour une réception savante.

Ainsi fut vendue à New York la grande photographie couleur Rhein II $(190 \times 360 \mathrm{~cm})$ réalisée en 1999 par l'auteur allemand Andreas Gursky. Le Rhin coule sous un ciel gris entre des champs verts. La taille d'un tel tirage photographique couleur relevait, à l'époque, d'une réelle performance, mais il convenait surtout de savoir qu' une usine avait été effacée, qu'elle avait disparu. La photographie n'était plus la trace des éléments du paysage mais l'inscription d'une absence. Si l'œuvre de Gursky s'est vendue aussi chère (4,3 millions de dollars), c'est parce que, derrière une apparente banalité, se cache l'exercice endogénétique né d'une inspiration, d'une fantaisie, d'une compétence de l'auteur. Ici, l'endogénétisme se donne pour document exogénétique; autrement dit, l'auteur fait disparaître son propre travail au profit d'une pseudo-objectivité photographique. Il se moque ainsi de ces observateurs qui croient

4. Voir Genesis, no 40, «Photo-graphies », 2015. 
encore naïvement à l'empreinte photographique. Le mouvement va dans le sens inverse de ce qui fut remarqué en littérature, lorsque les éléments exogénétiques d'un avant-texte se fondent en une matière endogénétique offerte au lecteur.

En mêlant finement endogenèse et exogenèse, en jouant avec les croyances communes, la photographie permet les doubles lectures et facilite paradoxalement les jeux fictionnels. Le photographe catalan Joan Fontcuberta est l'un de ceux qui jouent de cette fausse réalité. Ainsi les inventions végétales d'Herbarium (1984), les chimères animales de Fauna (1985-1989) et les paysages en trois dimensions d'Orogenesis nés non de cartes géographiques mais de peintures d'artistes.

Plus classiquement, l'auteur peut partir en quête d'informations ou de documents exogénétiques. Cela n'est pas une surprise pour la critique génétique. À l'instar d'une écriture littéraire sont mis en œuvre des repérages, des carnets de terrain, des correspondances, des écrits d'accompagnement... Ils s'accompagnent de polaroïds de repérage, de négatifs, de contacts, de tirages d'essais, de tirages de presse, de citations photographiques, d'autocitations (notamment dans les montages photographiques)... Le GPS, Google map et les cartes routières, les cartes d'état-major sont aujourd'hui les outils communs d'un juste repérage topographique.

Il faut reconnaître que ce qui importe pour l'auteur est autant l'acte photographique - l'action de prise de vue, le travail en studio de la retouche et du tirage - que l'image elle-même. Un photographe tel Bernard Plossu ne quitte pas son Rolleiflex, toujours prêt à saisir le moindre hasard, la moindre rencontre. Ce qu'il photographie n'est pas le désert, ni le Stromboli, ni même ses enfants ou ses amis, mais bien la scène photographique dont il est lui-même partie prenante, au cœur de laquelle il vit un moment intense; une scène qui attise le regard, convoque l'imagination, mobilise le corps, met les sens en relation avec les objets, les êtres rencontrés. Une scène qui l'implique pleinement et dont il conservera, à vie, la mémoire.

Cette analyse des photographies de Bernard Plossu permet non seulement de saisir pleinement le sens de ce que certains considèrent comme des « défauts »- horizon oblique, flous, décadrages, taches de lumière -, mais encore de comprendre et d'apprécier pleinement les travaux des artistes photographes. Nous sommes loin ici d'une photographie-empreinte.

Fig. 1 : Bernard Plossu, Shane, Santa Fe, New Mexico, 1983.

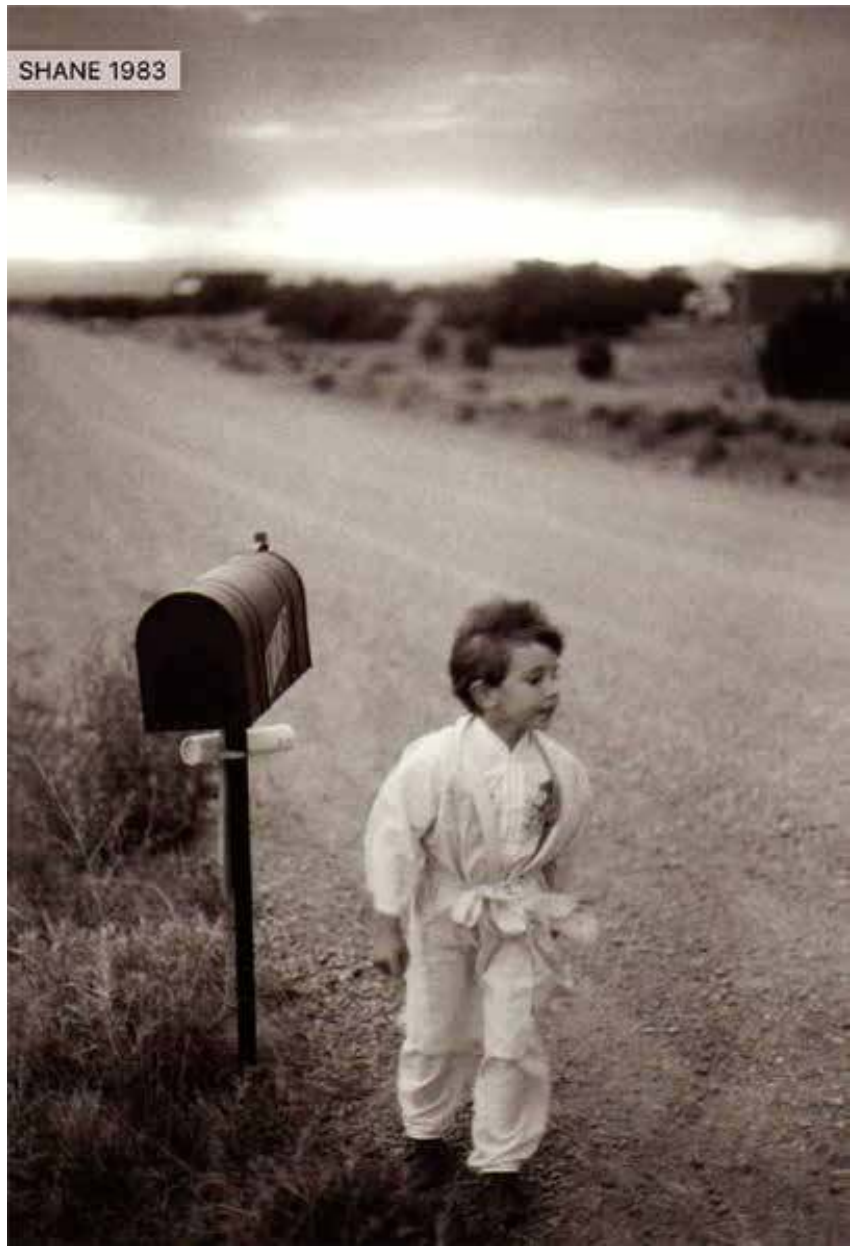

(C) Bernard Plossu 
Cependant, les négatifs, les contacts, les fichiers numériques s'accumulent. Ils nécessitent très vite une indexation, un archivage de qualité. Le photographe se plongera plus tard, de nouveau, dans ses planches-contacts, ses tirages de travail et redécouvrira, à l'occasion, l'intérêt oublié d'une photo.

L'élagage, étape de sélection drastique des négatifs à tirer, des fichiers à conserver, relève du travail endogénétique. Il n'est pas rare qu'il atteigne 90 ou $95 \%$ du total des prises de vues. Ce chiffre s'accroît encore en photographie numérique. La rétraction de la masse dont l'importance caractérise la création photographique, peut cependant avoir lieu à toute étape du travail, de la prise de vue à la publication.

\section{L'original en question}

La photographie est porteuse d'une extraordinaire tension entre exogenèse et endogenèse qui en est le fondement même. Nier la première serait nous priver tant de la marche du monde que de la vie citoyenne ou quotidienne, de l'archive, de la construction de l'histoire. Sous-estimer la seconde serait réduire la photographie à un automatisme, anéantir les auteurs, méconnaître le sens qu'ils accordent à leur œuvre. Ce serait aussi nier la part d'écriture à laquelle invite ce médium remarquable.

La tension entre exogenèse et endogenèse, entre document et création personnelle, entre écriture singulière et banalité objective peut conduire au conflit entre l'auteur et les utilisateurs de l'image. Le Code de la propriété intellectuelle reconnaît la photographie d'auteur comme œuvre d'art à la condition que le tirage soit limité à trente exemplaires, tous formats confondus, numérotés et signés par l'auteur. Malgré cela, certains jugements jouent en défaveur de l'auteur lorsqu'ils estiment que «le genre documentaire ne fait pas l'objet d'une protection au titre du droit d'auteur». La cour est, dès lors, en droit de demander au photographe - et cela image par image - ce qu'il a «ajouté à la réalité en introduisant des choix personnels». La simple sélection des options de point de vue, de cadrage, de focale, de saturation des couleurs... ne suffit pas. Au savoir-faire doit s'ajouter le reflet d'une approche personnelle, la touche d'originalité. Et lorsque le juge ne peut dire de quelle manière elle se marque dans l'image, le photographe réclamant l'autorat peut être débouté de sa plainte.

L'ambiguïté règne. En toute logique, pourraient être strictement relégués dans une aventure exclusivement exogène tout travail de photojournalisme $-\mathrm{y}$ compris lorsque la vie du photographe est mise en jeu -, les photographies initiales et reconductions des Observatoires de l'image, les photographies officielles des présidents de la République, l'œuvre entier d'un Henri Cartier-Bresson ou d'un Raymond Depardon - si n'opérait pas ici le poids injuste de la notoriété - et finalement toutes les œuvres travaillées, sophistiquées, lorsqu'elles ne sont pas numérotées, les portraits lorsque la personne photographiée n'a pas produit un document signé montrant qu'elle «accepte que l'auteur la photographie».

Ces remarques trahissent une méconnaissance largement répandue du travail de l'artiste photographe comme du concept d'œuvre photographique. La génétique et l'étude précise des processus de création s'avèrent ici d'un grand secours : en informant sur les processus de création et sur les intentions de l'artiste, elles permettent de s'arracher aux incertitudes de l'analyse de l'image achevée, à celles de la recherche insensée des critères d'originalité. 
La génétique littéraire s'est érigée sur la notion de «manuscrit moderne», sur l'existence de traces et sur la possibilité d'organiser celles-ci en états successifs d'un processus réalisé par un auteur. La question s'avère plus délicate en matière de génétique photographique.

Certes, la reconstitution génétique est favorisée par la préservation des négatifs, par l'existence de planches-contacts, par la conservation des fichiers numériques, par la présence d'écrits liés mais le cheminement créatif est rarement linéaire; les reprises, autocitations, recyclages sont nombreux. Une même photographie se retrouve dans plusieurs projets, parfois sous plusieurs aspects. Un même projet s'élabore à l'aide d'images réalisées dans des contextes et des temporalités différents. Un nouveau tirage s'effectue loin de la prise de vue et du tirage vintage... Il reste que les auteurs agissent en créateurs. Pour qui sait les recevoir, leurs images portent pleinement la trace de leurs choix, de leurs actions et de leur présence. 
Spécialiste d'histoire et de philosophie de la photographie, MONIQUE SICARD est réalisatrice de films documentaires et commissaire d'expositions. Ancienne élève de l'École normale supérieure, elle a dirigé au sein de l'ITEM (CNRS-ENS) l'équipe «Genèses des arts visuels », codirigé le programme «Photopaysage », et continue ses activités de recherche à l'Institut interdisciplinaire d'anthropologie du contemporain (CNRS-EHESS). Elle a publié récemment avec Malvina Borgherini PhotoPaysage, Il paesaggio inventato dalla fotografia, Quodlibet Studio, Italie, 2020; avec Aurèle Crasson et Gabrielle Andries La Fabrique photographique des paysages, Hermann, Paris, 2017; Genesis, no 40, «Photo-graphies », PUPS, Paris, 2015.

sicard.monique@gmail.com

Résumés

Exogenèse, endogenèse photographiques

La photographie apparaît porteuse d'une indicialité fondatrice et essentielle : elle prélèverait un morceau du monde. Dans l'imaginaire commun, collectif, cette exogénèse prime sur l'endogénèse, l'auteur disparaissant au profit de la chose représentée. Ainsi, la photographie a façonné, dès le début du XIXe siècle, un espace remarquable, «objectif», dont le sujet est exclu. Et cependant, l'acte photographique n'est jamais réductible à l'incorporation directe, automatique, de la réalité. Ce sont les gestes d'écriture qui lui donnent sens. En mêlant finement endogénèse et exogénèse en un véritable métissage, l'auteur photographe joue avec les croyances qui visent à ignorer l'auteur. L'acte créatif s'enrichit paradoxalement en retour, favorisant les jeux fictionnels et les doubles lectures. L'auteur, invisible, tire les ficelles du jeu.

Photography carries a fundamental and essential indexicality: it captures a piece of the world. In the collective imagination, exogenesis takes precedence over endogenesis, and the author disappears in favor of the thing represented. From the beginning of the $19^{\text {th }}$ century, photography has shaped a remarkable, "objective" space from which the subject is excluded. And yet, the act of photography is never reducible to the direct, automatic incorporation of reality. It is writing that gives meaning to photography. By deftly combining endogenesis and exogenesis in a truly integral way, the author-photographer plays with beliefs that aim to ignore the author. The creative act is paradoxically enriched in return, favoring fictional games and double readings, the invisible author pulling the strings behind the scenes.

Die Fotografie scheint eine grundlegende und wesentliche Indizialität in sich zu tragen: Sie soll ein Stück der Welt erobern. Im gemeinsamen, kollektiven Imaginären hat diese Exogenese Vorrang vor der Endogenese, wobei der Autor zugunsten des Dargestellten verschwindet. So hat die Fotografie seit Beginn des neunzehnten Jahrhunderts einen bemerkenswerten „objektiven“ Raum geschaffen, aus dem das Subjekt ausgeschlossen ist. Und doch lässt sich der fotografische Akt niemals auf die direkte, automatische Einverleibung der Wirklichkeit reduzieren. Es sind die Gesten des Schreibens, die ihm Bedeutung verleihen. Indem er Endogenese und Exogenese in einer echten Kreuzung fein vermischt, spielt der Autor-Fotograf mit Überzeugungen, die darauf abzielen, den Autor zu ignorieren. Der schöpferische Akt bereichert sich im Gegenzug paradoxerweise selbst, indem er fiktionale Spiele und doppelte Lesarten begünstigt. Der Autor, unsichtbar, zieht die Fäden des Spiels.
La fotografía conlleva aparentemente una indicialidad fundadora y esencial: extrae un fragmento del mundo. En el imaginario común, colectivo, esta exogénesis prevalece sobre la endogénesis, el autor desaparece en beneficio de lo representado. De este modo, la fotografía ha forjado desde comienzos del siglo XIX un espacio excepcional, "objetivo, cuyo sujeto resulta excluido. Sin embargo, el acto fotográfico no puede en ningún caso reducirse a la incorporación directa, automática, de la realidad. Son los gestos de escritura los que le otorgan un sentido. Mezclando finamente endogénesis y exogénesis en un verdadero mestizaje, el autor fotógrafo juega con las creencias que buscan ignorar al autor. En contrapartida, el acto creativo se enriquece paradójicamente, al favorecer los juegos ficcionales y las lecturas dobles. El autor, invisible, mueve los hilos del juego.

A fotografia tem uma indicialidade fundadora e essencial: capta um pedaço do mundo. No imaginário comum, coletivo, esta exogénese prevalece sobre a endogénese, desaparecendo o autor a favor da coisa representada. Assim, a fotografia moldou, desde o início do século XIX, um espaço notável, "objetivo", do qual o sujeito está excluído. No entanto, o ato fotográfico nunca é redutível à incorporação direta, automática, da realidade. São os gestos de escrita que lhe dão sentido. Ao misturar subtilmente endogénese e exogénese num verdadeiro cruzamento, o autor fotógrafo joga com as crenças que visam ignorar o autor. Em contrapartida, o ato criativo enriquece-se paradoxalmente, favorecendo jogos ficcionais e leituras duplas. O autor, invisível, manipula o jogo.

La fotografia porta con sé un'indizialità fondatrice ed essenziale: essa preleva un pezzo di mondo. Nell'immaginario comune, collettivo, questa esogenesi prevale sull'endogenesi, poiché l'autore sparisce a vantaggio della cosa rappresentata. Così, fin dall'inizio del diciannovesimo secolo, la fotografia ha plasmato uno spazio eccezionale, "obiettivo", dal quale il soggetto è escluso. E tuttavia l'atto fotografico non si può mai ridurre alla sola incorporazione diretta e automatica della realtà; sono i gesti di scrittura che gli danno senso. Unendo finemente endogenesi ed esogenesi in una vera e propria ibridazione, l'autore fotografo gioca con le credenze che puntano a ignorare l'autore. L'atto creativo si arricchisce paradossalmente di rimando, favorendo i giochi finzionali e le doppie letture. L'autore, invisibile, tira le fila di questo gioco. 